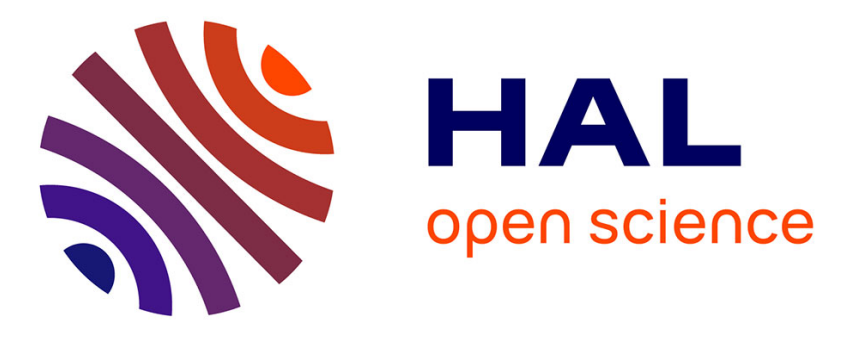

\title{
A non-linear viscoelastic model to describe the mechanical behavior's evolution of biodegradable polymers during hydrolytic degradation
}

Quentin Breche, Grégory Chagnon, Guilherme Machado, Benjamin Nottelet, Xavier Garric, Edouard Girard, Denis Favier

\section{To cite this version:}

Quentin Breche, Grégory Chagnon, Guilherme Machado, Benjamin Nottelet, Xavier Garric, et al.. A non-linear viscoelastic model to describe the mechanical behavior's evolution of biodegradable polymers during hydrolytic degradation. Polymer Degradation and Stability, 2016, 131, pp.145-156. 10.1016/j.polymdegradstab.2016.07.014 . hal-01369282

\section{HAL Id: hal-01369282 https://hal.science/hal-01369282}

Submitted on 9 Nov 2018

HAL is a multi-disciplinary open access archive for the deposit and dissemination of scientific research documents, whether they are published or not. The documents may come from teaching and research institutions in France or abroad, or from public or private research centers.
L'archive ouverte pluridisciplinaire HAL, est destinée au dépôt et à la diffusion de documents scientifiques de niveau recherche, publiés ou non, émanant des établissements d'enseignement et de recherche français ou étrangers, des laboratoires publics ou privés. 


\title{
A non-linear viscoelastic model to describe the mechanical behaviour's evolution of biodegradable polymers during hydrolytic degradation
}

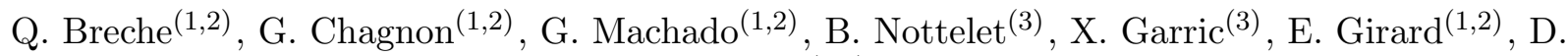 \\ Favier $^{(1,2)}$
}

(1) Université Grenoble-Alpes, TIMC-IMAG, F-38000 Grenoble France.

(2)CNRS, TIMC-IMAG, F-38000 Grenoble France.

(3) Université de Montpellier, Faculté de pharmacie/Institut des biomolécules Max Mousseron

(IBMM)/CNRS/UMR5247, 34093 Montpellier, France,

The biodegradable triblock copolymer PLA- $b$-PEG- $b$-PLA presents, in its initial state, a non-linear viscoelastic behavior. Its mechanical properties evolves during the in vitro degradation process. Tensile and relaxation tests are performed at $2 \%, 4 \%$ and $6 \%$ of load strain for different degradation steps. In order to describe the behavior of the polymer during degradation, an adaptive quasi-linear viscoelastic model is considered. In a first step, the model calibrated on the non-degraded state, perfectly fits the load and relaxation curves for every strain. Then, based on considerations about the preservation of the normalized relaxation curves over degration time, the adaptive quasi-linear viscoelastic model is adapted to degradation. A degradation parameter that drives the mechanical degradation kinetics is deduced for every tested degradation states. A physically motivated model is finally used to describe the degradation parameter at every degradation step. The whole constitutive model is very accurate to fit the mechanical curves at every strain during degradation.

Keywords: PLA-b-PEG-b-PLA, Mechanical behaviour's evolution, Biodegradable, Viscoelasticity, Mechanical model

\section{Introduction}

Due to its large range of mechanical properties and degradation times, the biodegradable and biocompatible tribloc copolymer PLA- $b$-PEG- $b$-PLA is an excellent candidate for tissue engineering applications [1]. In this case, the polymer is used to be a physical temporary support for the tissular reconstruction called "scaffold". The objective of this scaffold is to be a substitute to injured tissues during healing and to provide a three dimensionnal environment for tissue reconstruction. Moreover, in many cases, the scaffold has to temporarily replace the functions of the tissues, especially the mechanical support. Thus, it is necessary to have a detailed knowledge of the initial mechanical properties and their evolution due to degradation. Therefore, in order to design a polymeric architecture for scaffolds, the development of mechanical constitutive equations that are able to model the initial mechanical properties and that take into account the degradation is essential.

In their initial state, the PLA-based biodegradable materials can present some complex nonlinear behaviors, especially non-linear viscoelasticity [2]. In order to model these properties, a 
large class of non-linear viscoelastic models used to describe the mechanical properties of polymeric biomaterials and living tissues is based on single integral models. These models are extensions of the linear viscoelastic model [3]. Pipkin and Rogers [4] developed a single integral model that can take into account the dependence on strain of stress relaxation modulus. By considering that strain and time variables can be separated, Fung [5] simplified the Pipkin and Rogers model. In his model, the strain of the linear single integral model is replaced by a non-linear function of strain called "instantaneous elastic response". Nevertheless, the model does not take into account the strain dependence of the time-related response and can be difficult to calibrate [6]. In order to overcome these problems, Nekouzadeh et al. [7] developed an alternative approach called "adaptive quasilinear viscoelastic" model that allows to simplify calibration and considers the strain non-linearity of the viscoelastic part.

The properties of PLA-based polymers are changing in use due to degradation. Their main degradation mechanism is the hydrolysis of ester links that breaks the polymeric bonds. It means that, in an aqueous media, a decrease of number average molecular weight, a mass loss and a material erosion can be observed [8]. Another important macroscopic effect of polymeric bonds cut is the lost of mechanical properties. Many studies show that Young's modulus and tensile strength decrease after a variably long latency period [9-11]. Thus, to predict the evolution of the mechanical properties of scaffolds, the constitutive equation must include hydrolytic damage. Two types of methods can be distinguished to model mechanical properties degradation. The first one consists in modelling the evolution of mechanical parameters such as Young's modulus or tensile strength by a micro-mechanical approach. The entropy spring theory [12] or the molecular dynamic approach [13] are used to model the Young's modulus evolution. By considering scissions as cavities in the polymeric matrix, Samami and Pan [14] modelled the polymer as a continuum solid containing randomly distributed cavities. Then, using existing theories for porous solids, they deduced a constitutive equation that predicts the evolution of Young's modulus and tensile strength. The second method consists in adapting classical hyperelastic [15], viscoelastic models [16] or viscoplastic model [17] to degradation by assuming that the model constitutive material parameters are dependent of a degradable variable [2]. These models are only calibrated on simple uniaxial load tensile tests. Breche et al. [18] performed uniaxial load-relaxation tests and shew that the normalized relaxation function can be considered as an invariant of the degradation. Thus, in the linear viscoelasticity formalism, they expressed the degradation variable as a function of a proportionnality coefficient between the curves at different steps of degradation. Their model permits to describe the mechanical behavior for small deformation i.e. until $2 \%$.

The objective of this paper is to establish a mechanical constitutive equation that is able to fit the PLA- $b$-PEG- $b$-PLA uniaxial behavior upon a strain of $2 \%$. To achieve this, a non-linear viscoelastic adaptive quasi-linear viscoelastic (AQLV) model that takes into account the hydrolytic degradation is developed. First, after presenting materials and methods, experimental results of load-relaxation tests on PLA- $b$-PEG- $b$-PLA samples are presented. In a second part, the formalism of the AQLV model is introduced. Then, the calibration and the efficiency of the model to fit the experimental data are presented for the non-degraded material. In a last part, the AQLV model is adapted to take into account the hydrolytic degradation by a hydrolytic damage variable. The ability of this model to fit degraded experimental data is finally discussed. 


\section{Materials and methods}

\subsection{Material}

The material used in this study is the same as the one used in a previous study [18]. The details about the elaboration of the material are related here. Poly(ethylene glycol) (average Mn $20000 \mathrm{~g} / \mathrm{mol}$ ), tin(II) 2-ethylhexanoate ( $\mathrm{Sn}(\mathrm{Oct}) 2,95 \%$ ), dichloromethane (DCM), diethyl ether and tetrahydrofuran (THF) were purchased from Sigma-Aldrich (St-Quentin Fallavier, France). D,L-lactide (D,L-LA) was purchased from Purac (Lyon, France).

PLA50-b-PEG-b-PLA50 triblock copolymer was synthesized following a procedure previously described by Leroy et al. [11]. Typically, predetermined amounts of D,L-LA and PEG were introduced in a flask. $\mathrm{Sn}$ (Oct)2 (0.1 molar \% with respect to LA units) was then added. After degassing, the flask was sealed under vacuum and polymerization was allowed to proceed at $110^{\circ} \mathrm{C}$. After 5 days, the copolymer was recovered by dissolution in DCM and precipitation in cold diethyl ether. Finally, the product was dried under reduced pressure to constant mass. The copolymer was obtained with a yield of $90 \%$. Polymerization degree of each PLA block and molecular weight of the synthesized triblock copolymers were calculated using the following equations:

$$
\begin{aligned}
& D P_{P L A}(\%)=\frac{1}{2} \times \frac{D P_{P E G}}{\frac{E G}{L A}} \\
& M_{\text {ntribloc }}(\%)=2 \times\left(D P_{P L A} \times 72\right)+M_{n P E G}
\end{aligned}
$$

with EG/LA being the ratio of ethylene oxide and lactyl units calculated from ${ }^{1} \mathrm{H}$ NMR spectra. The polymer films have been manufactured by solvent evaporation. A predefined quantity of polymer was solubilized in acetone, spread in a small dish and placed during 36 hours under an extractor hood for evaporation. Final solvent removal was obtained by further drying in vacuo under $1.10^{-3} \mathrm{mBar}$ for 3 days. A $0.5 \mathrm{~mm}$ thick film was then obtained.

\subsection{Methods for mechanical tests}

Dogbone tensile specimens were cut in $0.5 \mathrm{~mm}$ thick films with a specific punch of $14 \mathrm{~mm}$ in length and $2 \mathrm{~mm}$ wide gauge length. They were then placed in $20 \mathrm{ml}$ test tubes and submitted to two times of degradation, 1 and 3 weeks, in phosphate buffer solution (PBS) at $37^{\circ} \mathrm{C}$ within an agitator/incubator. The mechanical tests were realized in an aqueous media at a controlled temperature of $37^{\circ} \mathrm{C}$ via a steel hermetic bath adjustable on the mechanical test machine in order to be in conditions as closed as possible to in vivo ones.

Uniaxial tensile tests on dogbone specimens at three different strain levels $(2 \%, 4 \%$ and $6 \%)$ with a strain rate during loading of $1 \%$ per second followed by a tensile relaxation test are performed. The latter were performed with a Gabo Eplexor mechanical test machine with a load cell of $10 \mathrm{~N}$ at 0,1 and 3 weeks of degradation. The strain field in specimens is measured by a Vic 2D image correlation software. At least two specimens were used for each test. The standard deviations were calculated and indicated on the $6 \%$ load curves by error bars as illustrated in figure 1). 


\section{Experimental data}

In a previous article [18], the mechanical tests at $2 \%$ and $4 \%$ have already been presented as well as mass lost, number average molecular weight, and dispersity index. This paper presents the results for larger strains i.e. $6 \%$. The figure 1 presents the stress-strain curves of the material for the tensile tests at a strain of $6 \%$ for every steps of degradation. In these curves, the strain is increasing until a strain of about $2 \%$ before reaching a plateau. The maximum stress reached in the non-degraded state is of $2.7 \mathrm{MPa}$. During degradation, the hydrolytic process cleaves the polymeric chains, leading to a decrease of molecular weight. It implies a decrease of the mechanical properties. As can be observed in figure 1, the maximum stress reached decreases even if the the general shape of the curve is conserved. After 3 weeks of degradation, about $20 \%$ of the inital non-degraded maximum stress is lost. The curve in 2 and 3 represent the load-relaxation tests for strains at $2 \%$ and $4 \%$ respectively and have already been presented in in a previous paper [18]. The figure 4 shows the stress recorded over time during the load-relaxation tests at a strain of $6 \%$. In this last figure, the load part corresponds to figure 1. After the loads, when strain is maintained constant, a stress relaxation is observed. It illustrates that the PLA- $b$-PEG- $b$-PLA is a viscoelastic material. A stress steady-state is reached after about 30 seconds, meaning that characteristic relaxation times are quite short. The residual stress was measured at $0.03 \mathrm{MPa}$ at $2 \%$ and at 0.08 $\mathrm{MPa}$ at $4 \%$ in paper [18] and are measured here at $0.25 \mathrm{MPa}$ at $6 \%$ in the non-degraded state. In order to compare the relaxation after the different degradation times, the normalized relaxation is used. It consists in the ratio of the current stress to the static stress before relaxation. If $\sigma_{m}$ is the static stress reached at the end of the load (corresponding to the dotted vertical lines in figures 2,3 and 4$)$ and $\sigma_{\text {relax }}$ the stress recorded during relaxation, the equation of the normalized relaxation is:

$$
\sigma_{n r}=\frac{\sigma_{\text {relax }}}{\sigma_{m}}=\frac{\sigma_{\text {relax }}}{\sigma_{\text {relax }}(t=0)}
$$

In this last equation, $t$ represents the time recorded during the stress relaxation. Thus, the initial time corresponds to the end of the load (represented by the dotted vertical lines in figure 2, 3 and 4). The normalized stress relaxation curves are presented in figure 5 for the different strains. One can observe that curves are not superimposed, meaning that the normalized stress relaxation of the material depends on the strain. This behavior is characteristic of the non-linear viscoelasticity.

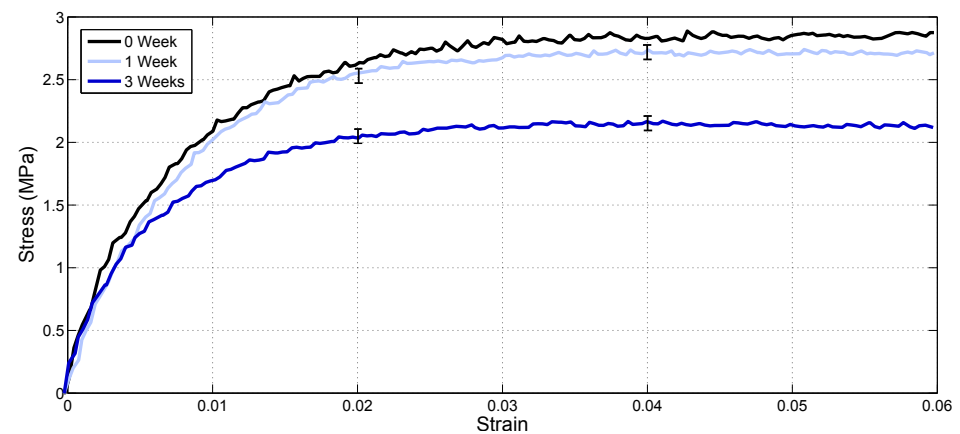

Figure 1: Experimental load curves at $6 \%$ strain load at 0, 1, and 3 weeks of degradation. 


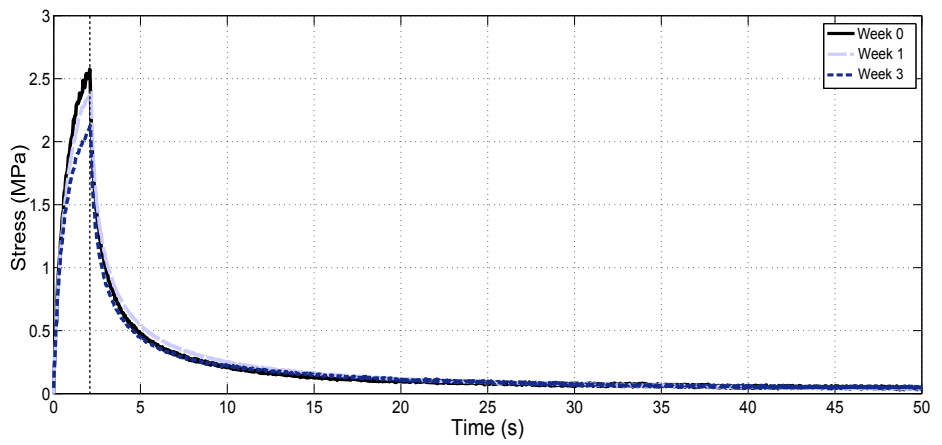

Figure 2: Experimental load and relaxation curves at $6 \%$ strain load at 0,1 , and 3 weeks of degradation.

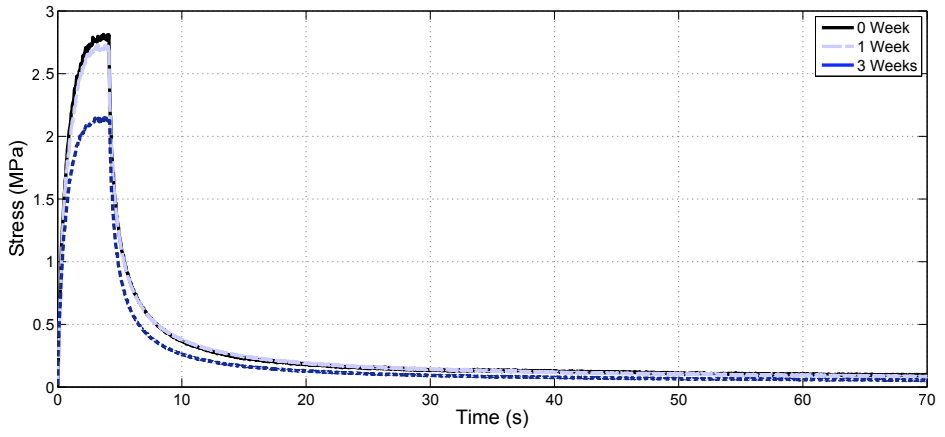

Figure 3: Experimental load and relaxation curves at $6 \%$ strain load at 0,1 , and 3 weeks of degradation.

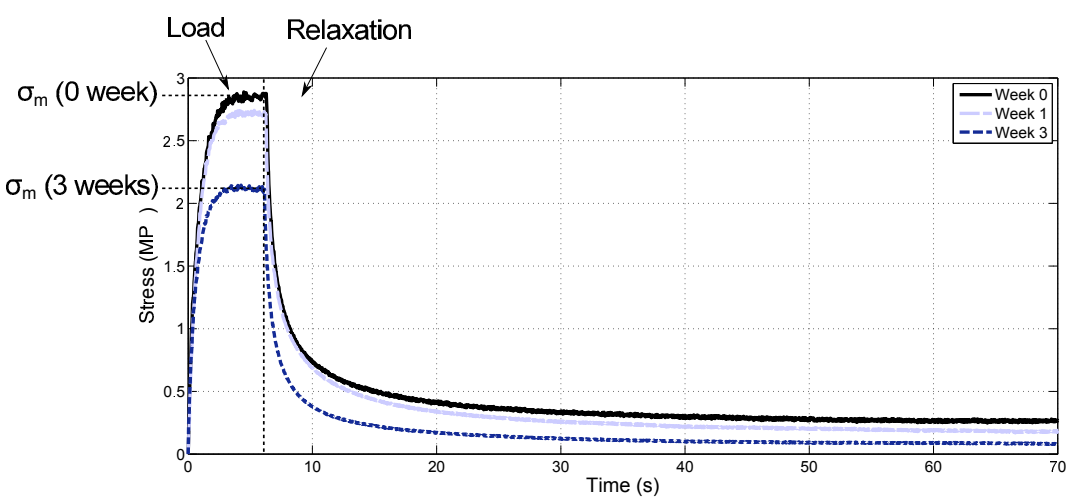

Figure 4: Experimental load and relaxation curves at $6 \%$ strain load at 0,1 , and 3 weeks of degradation. 


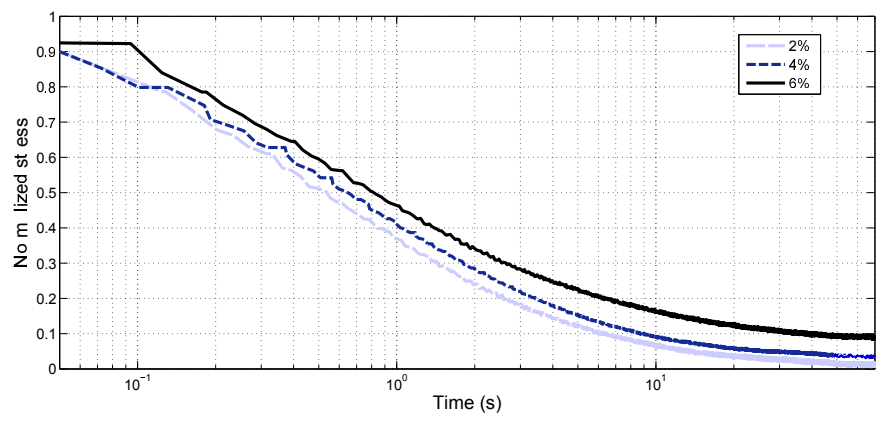

Figure 5: Normalized relaxation curves at 2\%, 4\%, and $6 \%$ load strain in the non degraded state.

\section{Constitutive modelling: an adaptive quasi-linear viscoelastic degradable model}

The aim of this section is to develop a viscoelastic degradable model that takes into account the non-linear viscoelasticity of the PLA- $b$-PEG- $b$-PLA and that allows to model its mechanical behaviour until a strain of $6 \%$.

\subsection{Hypothesis}

It is considered that the polymer is sufficiently hydrophilic to present a bulk erosion scheme and sufficiently thin to neglect autocatalytic effects [19]. Thus, the strain and degradation states are identical in every point of the specimens.

\subsection{Adaptive quasi-linear viscoelastic model}

In a previous study, Breche et al. [18] developed a simple generalized Maxwell model to predict the behaviour of PLA- $b$-PEG- $b$-PLA. The model was efficient for strain lower than $2 \%$. Nevertheless, it was unable to fit the experimental tests upon $2 \%$ strain and to take into account the strain dependence of the normalized relaxation function. In the previous paper, a linear viscoelastic Maxwell model was used. In order to improve the modelling, a non-linear viscoelastic model called "adaptive quasi-linear viscoelastic" (AQLV) is considered. In the general case of the AQLV model , the stress is equal to the product of a pure non-linear function $k(\varepsilon)$ and an intermediate variable called the "viscoelastic strain" $V^{\varepsilon}(t)[7]$, that is a linear convolution integral.

$$
\left\{\begin{array}{l}
\sigma(t)=k(\varepsilon(t)) V^{\varepsilon}(t) \\
V^{\varepsilon}(t)=\int_{0}^{t} g(t-\tau) \frac{d \varepsilon}{d \tau} d \tau
\end{array}\right.
$$

where $g(t)$ is a "reduced" relaxation function. To take into account the strain dependance of the normalized relaxation functions, degrees of freedom are added to the model by allowing different non-linear behaviours for different "reduced" relaxation functions as suggested by Nekouzadeh et al. [7]. 


$$
\left\{\begin{array}{l}
\sigma(t)=\sigma_{\infty}(\varepsilon(t))+\sum_{i} k_{i}(\varepsilon(t)) V_{i}^{\varepsilon}(t) \\
V_{i}^{\varepsilon}(t)=\int_{0}^{t} g_{i}(t-\tau) \frac{d \varepsilon}{d \tau} d \tau
\end{array}\right.
$$

$\sigma_{\infty}$ is a function of strain only representing the residual stress. The "reduced" relaxation functions are chosen to be decreasing exponential functions $g_{i}(t)=e^{-\frac{t}{\tau_{i}(\varepsilon)}}[7]$ where $\tau_{i}$ are characteristic relaxation times. Thus, by analogy with the previous article [18], the model can be represented in term of hypothetical parallel Maxwell elements. Its rheological scheme is presented in figure 6 . In this representation, spring stiffnesses $\left(k_{i}(\varepsilon)\right)$ and dashpot coefficients $\left(\eta_{i}(\varepsilon)\right)$ are functions of the overall strain. For each branch, it exists the following relationships:

$$
\left\{\begin{array}{c}
\dot{V}_{i}^{\varepsilon}+\frac{V_{i}^{\varepsilon}}{\tau_{i}(\varepsilon)}=\dot{\varepsilon} \\
\sigma_{i}=k_{i}(\varepsilon) V_{i}^{\varepsilon}
\end{array}\right.
$$

where :

$$
\tau_{i}(\varepsilon)=\frac{k_{i}(\varepsilon)}{\eta_{i}(\varepsilon)}
$$

To simplify, it is considered that the spring stiffnesses and dashpot coefficients are proportionnal to the same nonlinear function of strain $f(\varepsilon)$ [7], meaning that :

$$
\tau_{i}(\varepsilon)=\frac{k_{i}(\varepsilon)}{\eta_{i}(\varepsilon)}=\frac{k_{i} f(\varepsilon)}{\eta_{i} f(\varepsilon)}=\frac{k_{i}}{\eta_{i}}=\tau_{i}
$$

Thus, $\tau_{i}$, the characteristic relaxation times, are independent on strain.

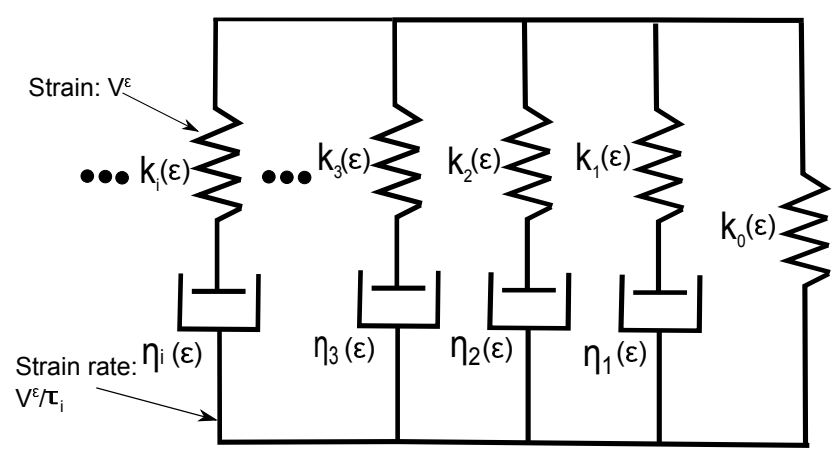

Figure 6: Rheological scheme of the model

\subsection{Calibration of the model on the non-degraded experimental data}

At first, the model is calibrated on the non-degraded experimental data. As seen in previous part (cf. figure 5), the non-linear character of the polymer behavior does not allow to consider the 
normalized relaxation as an invariant of the strain. Sarver et al. [20] proposed a normalization in the following system of stress coordinates:

$$
\sigma_{n r}^{*}=\frac{\sigma_{\text {relax }}^{*}}{\sigma_{m}^{*}}=\frac{\sigma_{\text {relax }}(\varepsilon)-\sigma_{\infty}(\varepsilon)}{\sigma_{m}(\varepsilon)-\sigma_{\infty}(\varepsilon)}=Q
$$

$Q$ is called the normalized relaxation factor. In figure 7, the normalized relaxation factors measured at strains of $2 \%, 4 \%$ and $6 \%$ are plotted. It can be observed that these curves are nearly similar. Thus, it is considered that it exists only one normalized relaxation factor master curve that is independent on strain.

Nekouzadeh et al. [7], determined the values of $k_{i}$ at every strain points for which a relaxation stress was measured. For this, a complex non-linear least square method that allows to fit simultaneously the relaxation curve at these different strains is used. From the obtained equations, the values of $k_{i}$ are deduced at these particular strain points. Then, the function $k_{i}(\varepsilon)$ is fitted from these points to obtain a value at every strain. Sarver et al. [20] shew that if the normalized relaxation factor is an invariant of strain, the calibration of the Fung's QLV model is greatly simplified. It is also the case for the AQLV model. It allows to determine, in a simple way, a value of $k_{i}(\varepsilon)$ at every experimental strain. At first, the equation of the master curve must be determined. To this end, the mean of the three normalized relaxation factors curves at strains of $2 \%, 4 \%$ and $6 \%$ is calculated. Then, this curve is fitted. Breche et al. [18] shew that for a PLA- $b$-PEG- $b$-PLA, the relaxation curve can be fitted with a three temporal terms Prony's serie. Thus, the master normalized relaxation factors curve is also fitted with three decreasing exponential functions:

$$
Q_{m}=\sum_{i=1}^{3} a_{i} e^{-\frac{t}{\tau_{i}}}
$$

where $a_{i}$ and $\tau_{i}$ are material parameters. The values of the parameters to fit the experimental data curves are presented in table 1 . The corresponding calculated relaxation curve is plotted in figure 7. Then, from the values obtained for the tests at $2 \%, 4 \%$ and $6 \%$, and by considering that the residual stress is equal to 0 in the absence of strain, the residual stress $\sigma_{\infty}(\varepsilon)$ is fitted as a function of the strain in order to obtain a value for every strain. Given the fact that four points need to be fitted, a three order polynomial function is chosen. Thus, $\sigma_{\infty}(\varepsilon)$ takes the form of a polynomial function of $\varepsilon, f(\varepsilon)$ :

$$
f(\varepsilon)=c_{0}+c_{3} \varepsilon^{3}+c_{2} \varepsilon^{2}+c_{1} \varepsilon
$$

$c_{i}$ are the coefficients of the polynomial function and their values are presented in table 2 . The fitted curve can be seen in figure 9 .

Table 1: Values of the coefficients of the polynomial functions of $k_{\varepsilon}$ and $\sigma_{\infty}$

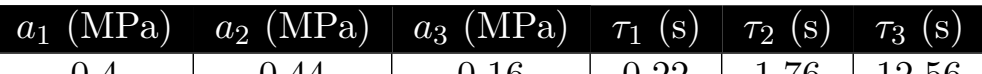

\begin{tabular}{|l|l|l|l|l|l|}
\hline 0.4 & 0.44 & 0.16 & 0.22 & 1.76 & 12.56 \\
\hline
\end{tabular}


Table 2: Values of the coefficients of the polynomial functions of $k_{\varepsilon}$ and $\sigma_{\infty}$

\begin{tabular}{|cc|c|c|c|}
\multicolumn{1}{c}{$k_{1}(\mathrm{MPa})$} & $k_{2}(\mathrm{MPa})$ & $k_{3}(\mathrm{MPa})$ & $\sigma_{\infty}(\mathrm{MPa})$ \\
$c_{0}$ & $2.08 \times 10^{2}$ & $1.50 \times 10^{2}$ & 49.30 & 0 \\
$c_{1}$ & $2.34 \times 10^{4}$ & $-4.34 \times 10^{3}$ & $-2.10 \times 10^{3}$ & 2.50 \\
$c_{2}$ & $-5.50 \times 10^{5}$ & $8.00 \times 10^{4}$ & $4.00 \times 10^{4}$ & -98.70 \\
$c_{3}$ & $4.07 \times 10^{6}$ & $-5.41 \times 10^{5}$ & $-2.72 \times 10^{5}$ & $2.10 \times 10^{3}$ \\
\hline
\end{tabular}

In the formalism of the model, the expression of the normalized relaxation factor is:

$$
Q(\varepsilon, t)=\frac{\sigma_{\text {relax }}(\varepsilon)-\sigma_{\infty}(\varepsilon)}{\sigma_{m}(\varepsilon)-\sigma_{\infty}(\varepsilon)}=\left(\sum_{i=1}^{3} k_{i}(\varepsilon) \tau_{i}\left(e^{\frac{T}{\tau_{i}}}-1\right) e^{-\frac{t}{\tau_{i}}}\right) \frac{1}{\sigma_{m}(\varepsilon)-\sigma_{\infty}(\varepsilon)} \times \frac{d \varepsilon}{d \tau}
$$

$\sigma_{m}(\varepsilon)$ represents the value of the stress in the load measured at a given strain and $T$ represents the duration of the load. Thus, from the values of $\sigma_{m}(\varepsilon)$, and the fitted equations of $\sigma_{\infty}$ and $Q_{m}$, the values of $k_{i}$ can be determined from equations (12) for every $\sigma_{m}(\varepsilon)$ measured and are presented in figure 8. As for the residual stress, the values of $k_{i}$ must be fitted to obtain an analytical expression of $k_{i}(\varepsilon)$ for every strain. In the same way, a three order polynomial function of the form of $f(\varepsilon)$ was determined. The coefficients of the function are presented in table 2 for every $k_{i}(\varepsilon)$. It perfectly fits the curves except for the first parts of $k_{2}$ and $k_{3}$ (cf. figure 8 and 9 ). As can be seen in figure 10 that presents the fitted load-relaxation curves and the corresponding experimental curves, the mechanical behavior is perfectly predicted for every strain. The lack of accuracy of the fitting for $k_{2}$ and $k_{3}$ at the beginning of the curves has thus no consequences on the ability of the model to represent experimental data.

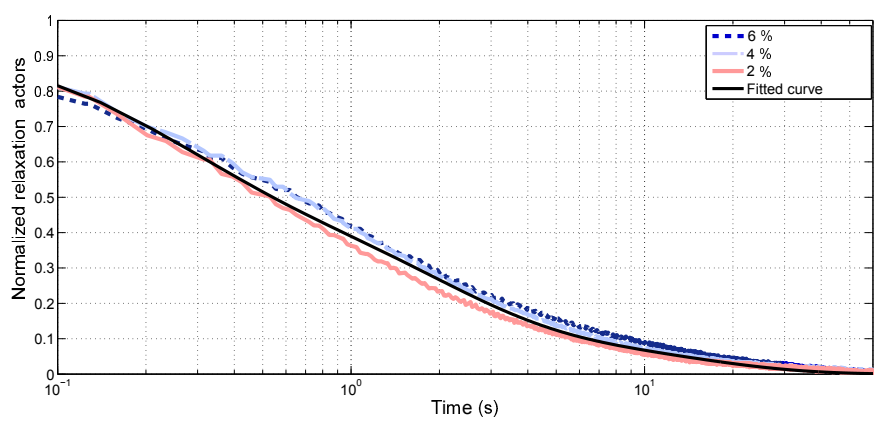

Figure 7: Normalized relaxation factors for different strain levels and the corresponding fitted master curve 

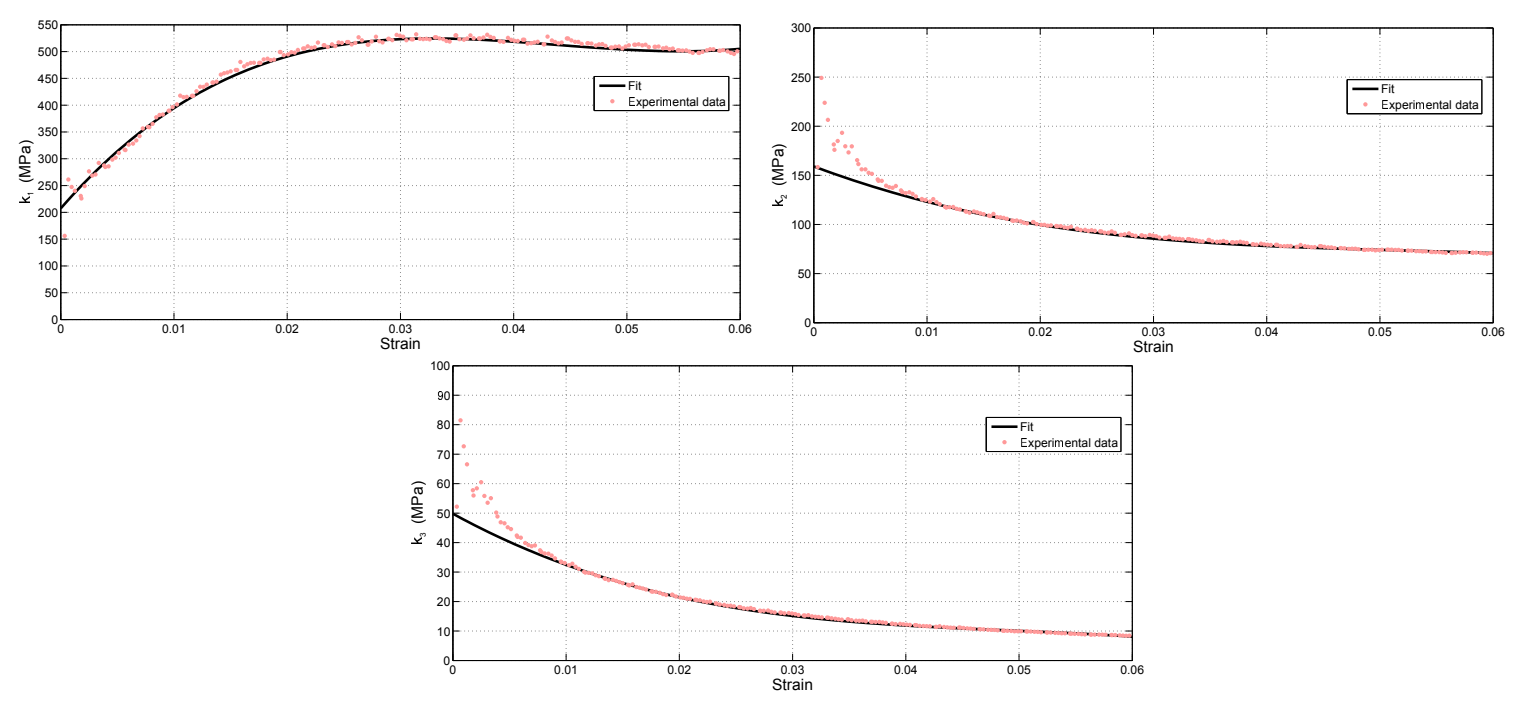

Figure 8: Values of the functions $k_{i}$ and their corresponding analytical fit

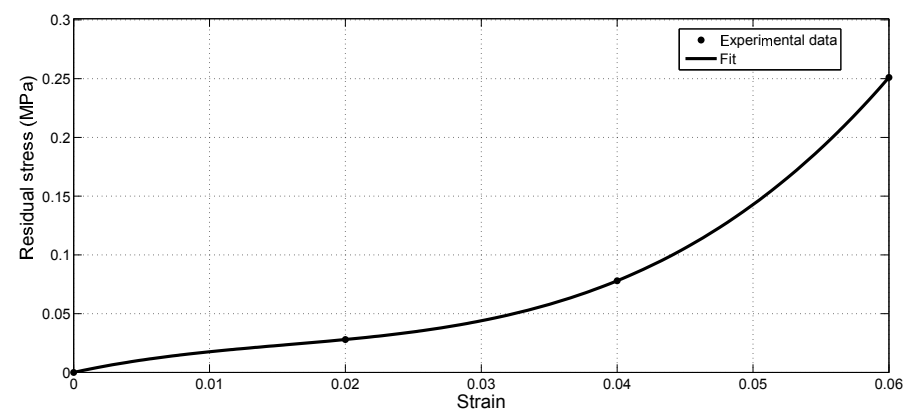

Figure 9: Residual stress for different strain levels 

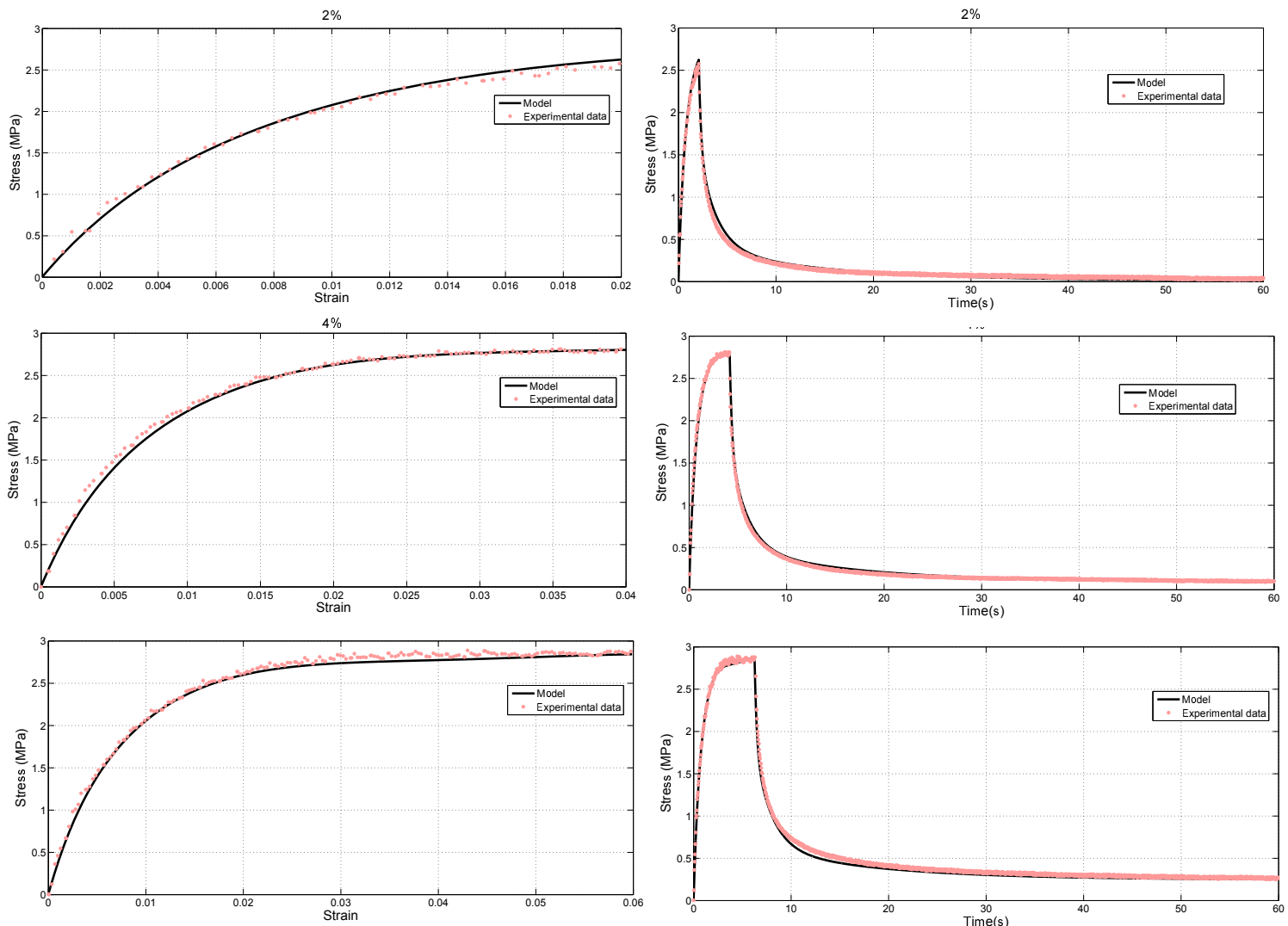

Figure 10: Experimental data versus calibrated model for different strain levels

\subsection{An AQLV degradable model based on the invariance of the normalized relaxation}

To take into account the evolution of mechanical properties during degradation, a scalar parameter $d_{h}\left(\boldsymbol{x}, t_{d}, \varepsilon\right)$ is introduced. It represents the degraded state of the polymer at each material point $\boldsymbol{x}$ and at a given degradation time $t_{d}[2]$. As seen in the hypothesis in part 4.1, $d_{h}$ is supposed independent of the location in the specimen. $d_{h}$ has a value between 0 and 1,0 representing a non-degraded state and 1 a fully degraded state.

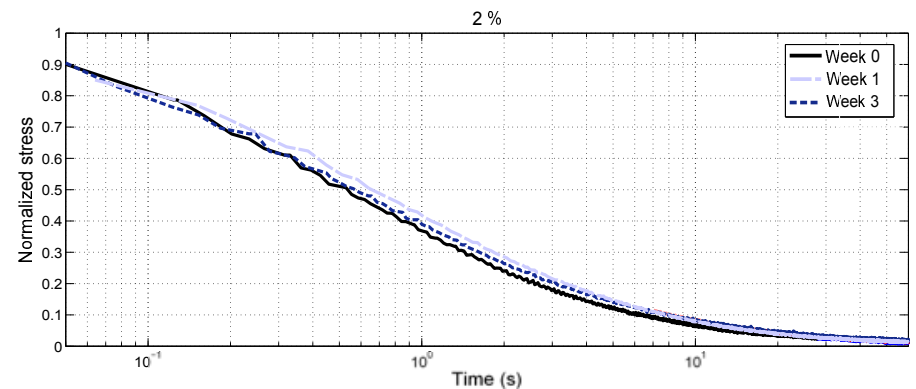

Figure 11: Normalized relaxations curve at a strain of $2 \%$ at different degradation steps 


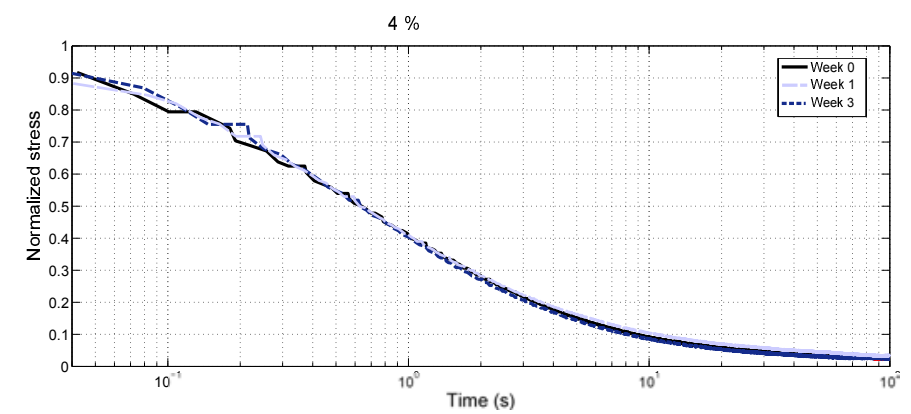

Figure 12: Normalized relaxations curve at a strain of $4 \%$ at different degradation steps

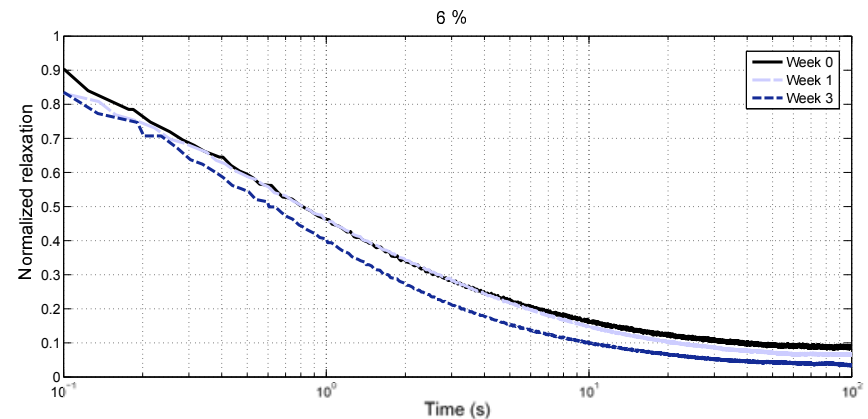

Figure 13: Normalized relaxations curve at at a strain of $6 \%$ at different degradation steps

The figures 11, 12 and 13 represent the normalized relaxation curves for $2 \%, 4 \%$ and $6 \%$ respectively at every step of degradation (calculated from the relaxation curves of the figures 2 , 3 and 4 and equation 3). For the two first strains, the normalized relaxation curves are perfectly superimposed, meaning that the temporal viscous component remains invariant during the three first weeks of degradation for these levels of strain. Nevertheless, at a strain of $6 \%$, a shift between the normalized relaxation curves is observed. At the third week of degradation, the residual stress of the normalized relaxation curves decreases. However, to simplify the model, it will be considered that the normalized relaxation curves, for every fixed value of strain, are independent on the degradation state. Thus, by admitting, at a given strain $\varepsilon$, that the non-degraded normalized relaxation curve is equal to the normalized relaxation curve at a degraded step, the equation of the degraded relaxation curve can be deduced. For this, equation 3 is used in considering that the relaxation stress depends on the degradation parameter and deformation.

$$
\sigma_{\text {relax }}\left(d_{h}, \varepsilon, t\right)=\frac{\sigma_{\text {relax }}\left(d_{h}, \varepsilon, t=0\right)}{\sigma_{\text {relax }}\left(d_{h}=0, \varepsilon, t=0\right)} \times \sigma_{\text {relax }}\left(d_{h}=0, \varepsilon, t\right)=\frac{\sigma_{m}\left(d_{h}, \varepsilon\right)}{\sigma_{m}\left(d_{h}=0, \varepsilon\right)} \times \sigma_{\text {relax }}\left(d_{h}=0, \varepsilon, t\right)
$$

In a load-relaxation test, $\sigma_{m}\left(d_{h}, \varepsilon\right)$ is, for a given strain $\varepsilon$ and a degradation state $d_{h}$, the stress 
reached at the end of the load and corresponds to the time zero of the relaxation. In the AQLV model, the equation of the stress relaxation at a given deformation is :

$$
\sigma_{\text {relax }}\left(d_{h}, \varepsilon, t\right)=\left(\sigma_{\infty}(\varepsilon)+\sum_{i=1}^{3} k_{i}(\varepsilon(t)) \times \frac{\sigma_{m}\left(d_{h}, \varepsilon\right)}{\sigma_{m}\left(d_{h}=0, \varepsilon\right)} \times \tau_{i}\left(e^{\frac{T}{\tau_{i}}}-1\right) e^{-\frac{t}{\tau_{i}}}\right) \frac{d \varepsilon}{d \tau}
$$

By combining equation 13 and 14 the general following equation can be obtained:

$$
\sigma_{\text {relax }}\left(d_{h}, \varepsilon, t\right)=\left(\sigma_{\infty}(\varepsilon) \times \frac{\sigma_{m}\left(d_{h}, \varepsilon\right)}{\sigma_{m}\left(d_{h}=0, \varepsilon\right)}+\sum_{i=1}^{3} k_{i}(\varepsilon(t)) \times \frac{\sigma_{m}\left(d_{h}, \varepsilon\right)}{\sigma_{m}\left(d_{h}=0, \varepsilon\right)} \times \tau_{i}\left(e^{\frac{T}{\tau_{i}}}-1\right) e^{-\frac{t}{\tau_{i}}}\right) \frac{d \varepsilon}{d \tau}
$$

From the definition of Lemaitre et al. [21] used by Vieira et al. [15] for hydrolytic damage, the degradation variable is defined as:

$$
d_{h}(\varepsilon)=1-\frac{\sigma_{m}\left(d_{h}, \varepsilon\right)}{\sigma_{m}\left(d_{h}=0, \varepsilon\right)}
$$

Consequently, the stress relaxation curve can be expressed as a function of $d_{h}$ :

$$
\sigma_{\text {relax }}\left(d_{h}, t\right)=\left(\sigma_{\infty} \times\left(1-d_{h}\right)+\sum_{i=1}^{3} k_{i}(\varepsilon(t)) \times\left(1-d_{h}\right) \tau_{i}\left(e^{\frac{T}{\tau_{i}}}-1\right) e^{-\frac{t}{\tau_{i}}}\right) \frac{d \varepsilon}{d \tau}
$$

Finally, from equation 5, the AQLV degradable model can be written as:

$$
\left\{\begin{array}{l}
\sigma(t)=\sigma_{\infty}(\varepsilon(t))\left(1-d_{h}\right)+\sum_{i} k_{i}(\varepsilon(t))\left(1-d_{h}\right) V_{i}^{\varepsilon}(t) \\
V_{i}^{\varepsilon}(t)=\int_{0}^{t} g_{i}(t-\tau) \frac{d \varepsilon}{d \tau} d \tau
\end{array}\right.
$$

From equation (16) the degradation parameters can be calculated as a function of strain for 1 and 3 weeks of degradation. The curves are presented in figure 14 and 15. In the first percent strain of the load, $d_{h}$ suffers important variations. It means that during this transitory part, the non-degraded and the degraded load curves are not perfectly proportionnal. Nevertheless, beyond this part, $d_{h}$ becomes almost constant, meaning that the shape of the curve is conserved in the course of degradation. In order to simplify the model, the hypothesis that $d_{h}$ is constant and independent on strain is used. To determine the value of $d_{h}$, the mean value between $1 \%$ and $6 \%$ is taken (black curves in figures 14 and 15). 


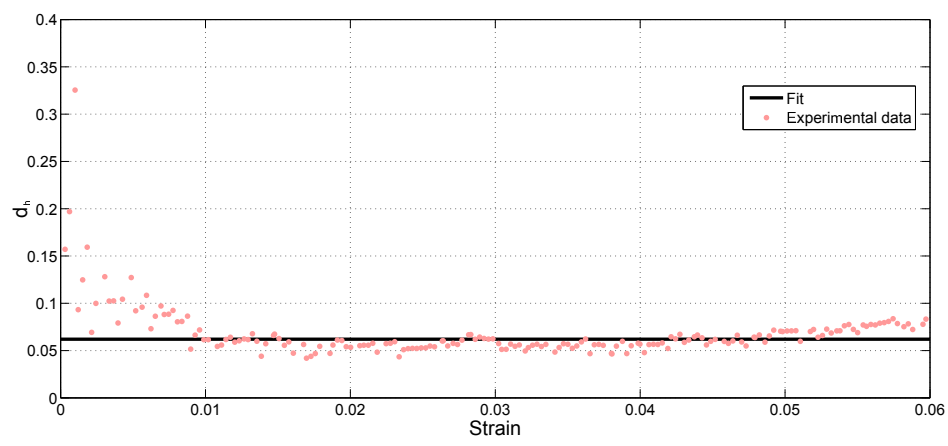

Figure 14: Degradation parameter as a function of strain after 1 week of degradation

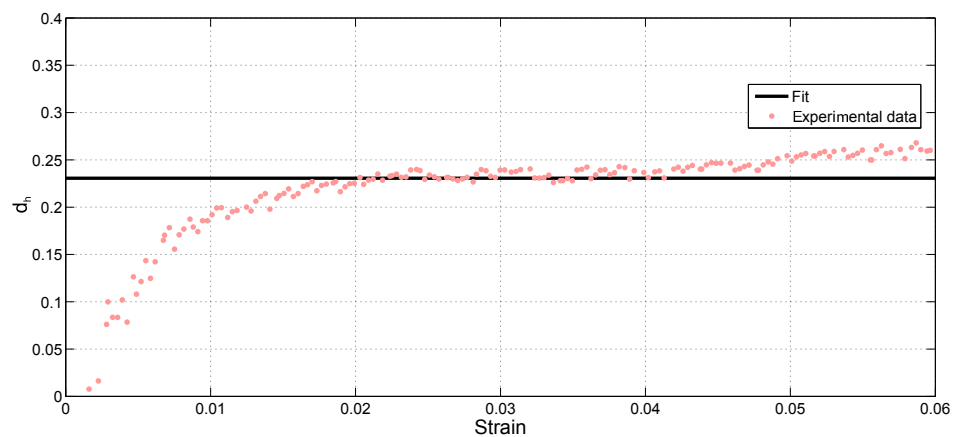

Figure 15: Degradation parameter as a function of strain after 3 weeks of degradation

When water diffuses into the polymeric structure, hydrolysis reaction breaks the ester links leading to the chains cleavage. This leads to the creation of oligomers and monomers. The main consequence is the decrease of the number average molecular weight and of the mechanical properties. It will be considered that the evolution of the degradation parameter is directly correlated with the decrease of the number average molecular weight [18]. In figure 16, the degradation parameter obtained from figures 14 and 15 is plotted as a function of the number average molecular weight for 0,1 and 3 weeks (black dots). In order to model the evolution of the degradation parameter for every intermediate step, the degradation parameter is fitted as a function of the normalized number average molecular weight. The expression proposed by Samami and Pan [14] for the evolution of tensile strength is used:

$$
d_{h}(\varepsilon)=1-\frac{\sigma_{m}\left(d_{h}, \varepsilon\right)}{\sigma_{m}\left(d_{h}=0, \varepsilon\right)}=1-\left(1-f_{v}\right)^{n}
$$

where $f_{v}\left(\frac{1}{m^{3}}\right)$ is the volume fraction of the cavities generated by polymer scissions. $f_{v}$ can be expressed, for early degradation times, as a function of the normalized number average molecular weight [14]. Thus, the expression of the degradation parameter becomes: 


$$
d_{h}=1-\left(1-K \times\left(\frac{M_{n 0}}{M_{n}}-1\right)\right)^{n}
$$

where $M_{n}$ is the actual number average molecular weight and $M_{n 0}$ is the initial number average molecular weight. $K$ is a fitting parameter that depends on the initial number average molecular weight, initial density, and radius of cavities created by scissions of the polymer. $n$ is also a fitting parameter. The values of these parameters are presented in table 3 . They are deduced from the measurement of the normalized number average molecular weight during degradation performed in a previous study [18]. The fitted function is presented in figure 16. In order to link the evolution's kinetics of normalized number average molecular weight to time, the following expression has been proposed previously [18]:

$$
\frac{M_{n 0}}{M_{n}}=1+K t
$$

where $K$ is a parameter that is proportionnal to the hydrolysis rate and equal to 0.62 in this case.

Table 3: Values of the parameters of the degradation variable kinetic model

\section{$\mathrm{K} \quad \mathrm{n}$}

$0.32 \quad 0.29$

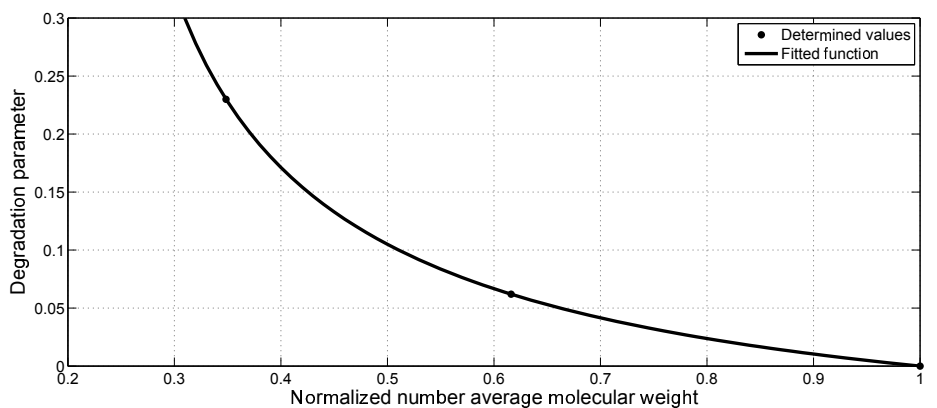

Figure 16: Degradation parameter's evolution fitting during degradation 

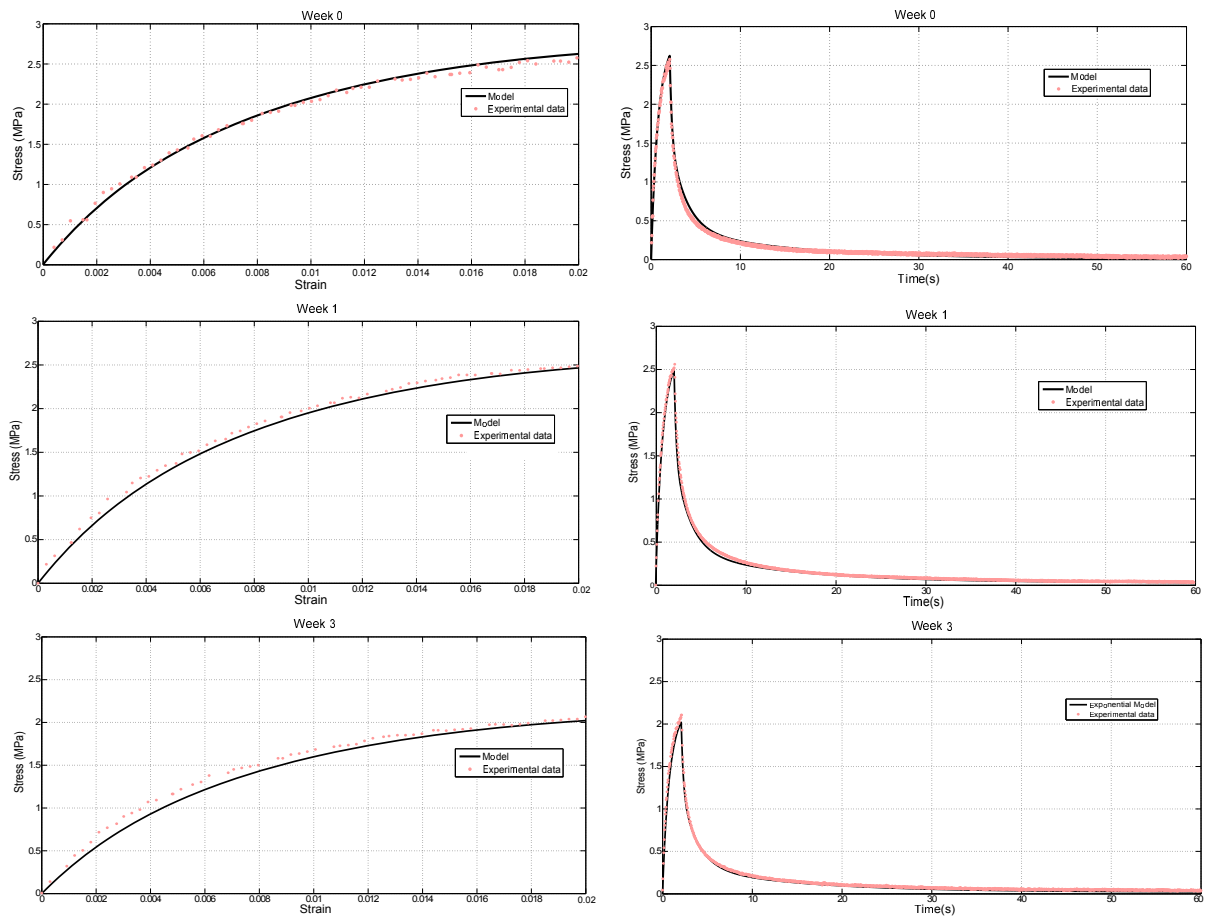

Figure 17: Experimental load relaxation tests (right) for 0, 1 and 3 weeks of degradation with corresponding load stress-strain curves (left) compared with calibrated AQLV model on the $2 \%$ load strain's tests. 

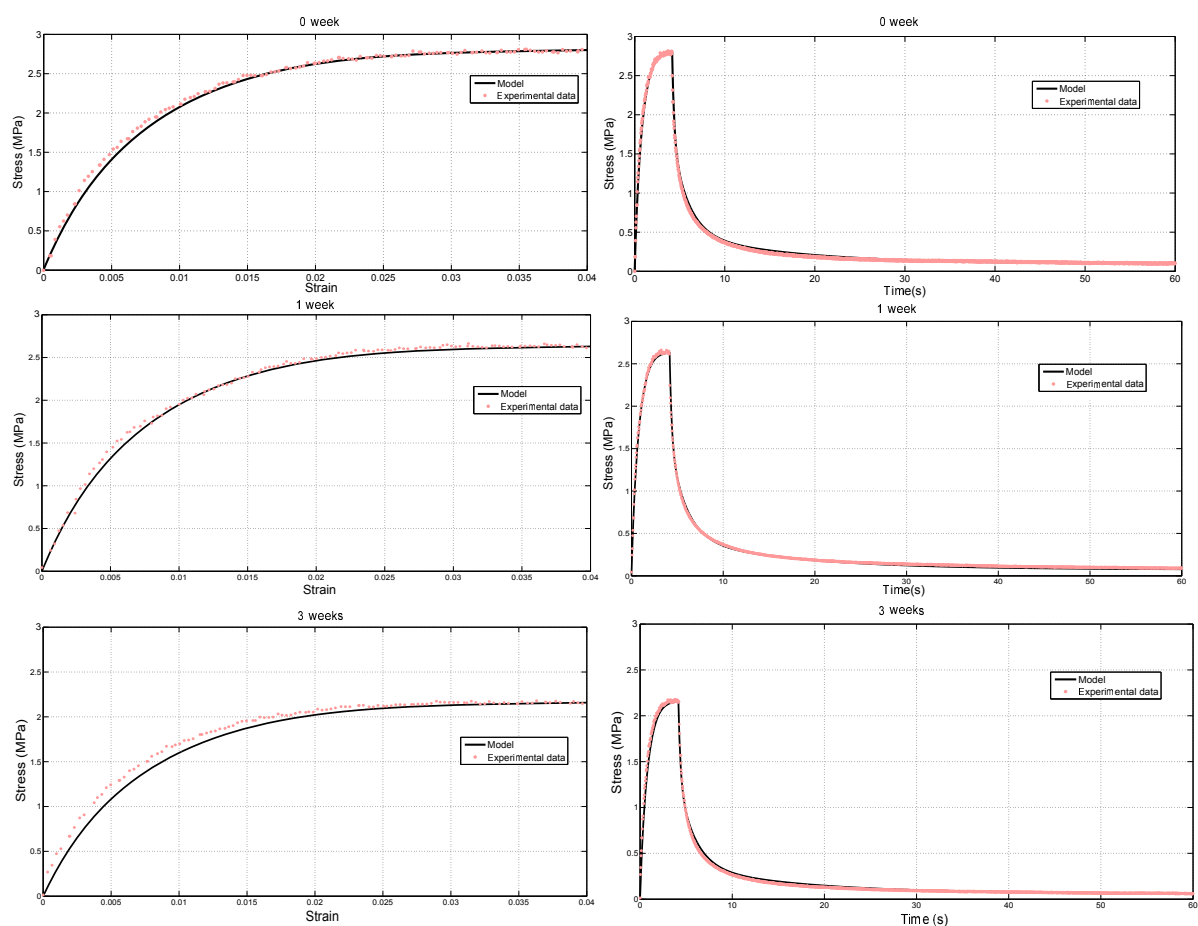

Figure 18: Experimental load relaxation tests (right) for 0,1 and 3 weeks of degradation with corresponding load stress-strain curves (left) compared with calibrated AQLV model on the $4 \%$ load strain's tests. 

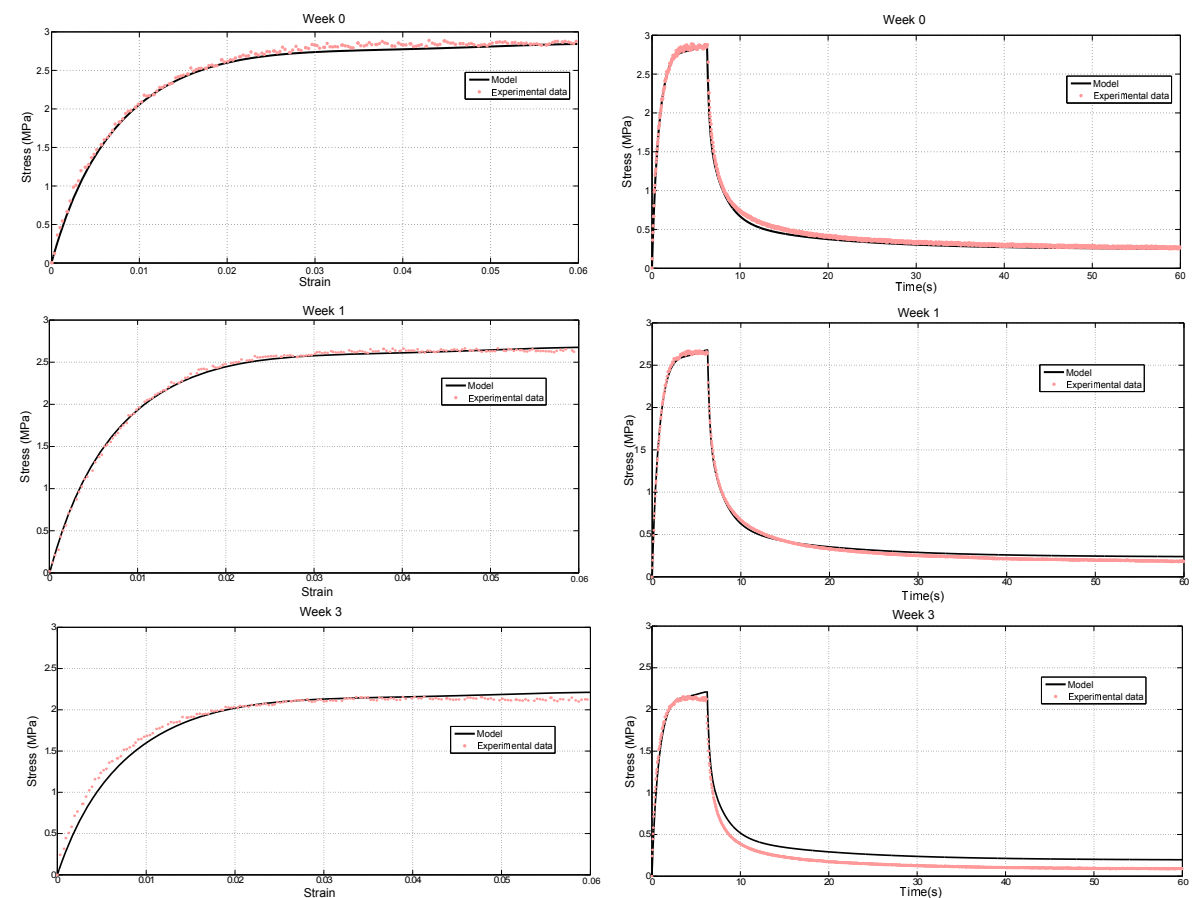

Figure 19: Experimental load relaxation tests (right) for 0, 1 and 3 weeks of degradation with corresponding load stress-strain curves (left) compared with calibrated AQLV model on the $6 \%$ load strain's tests.

As can be seen in figures 17 and 18 the model perfectly fits both load and relaxation curves at strains of $2 \%$ and $4 \%$ for every week of degradation. At $6 \%$ strain, the model is efficient to predict the mechanical behavior for 0 and 1 week of degradation as can be observed in figure 19. Nevertheless, it starts to be less accurate for the third week of degradation at $6 \%$ strain. The beginning of the load part is a bit underestimated and the end overestimated. This result was expected and is due to the approximation that the degradation parameter is constant for every strain, that is not totally the case at three weeks of degradation (cf. figure 15). At this degradation and strain states, the relaxation curve is not perfectly fitted. Indeed, the residual stress is overestimated and its value is twice times larger than the experimental one. It can be explained by the fact that the hypothesis of the invariance of the normalized relaxation function during degradation is not totally verified. It can be seen in figure 13 that at $6 \%$, the normalized relaxation functions are not exactly the same, especially for 3 weeks of degradation.

\section{Conclusions}

In order to predict the behavior of a PLA- $b$-PEG- $b$-PLA biodegradable polymers during degradation, a non-linear viscoelastic model that takes into account the hydrolytic degradation during the three first weeks of degradation was developed. From an experimental protocol of load-relaxation tests, the non-linear viscoelastic behavior of the PLA- $b$-PEG- $b$-PLA was highlighted. An adaptive quasi-linear viscoelastic model was then calibrated on the non-degraded tests. The ability of the model to describe the uniaxial load-relaxation behavior of the non-degraded polymer was demonstrated for every strain lower than $6 \%$. By using the normalized relaxation function invariance 
during degradation, the adaptive quasi-linear model was adapted to take into account the degradation through a hydrolytic degradation parameter. Then, a physical kinetics evolution of this parameter was used to model every degradation times between zero and three weeks. The model is compared to experimental data for every degradation step and strain. It was shown that the model fits very well the experimental data. Nevertheless, in the most degraded case, the relaxation curve that appears at the load at $6 \%$ is not perfectly fitted. This could be improved by choosing the normalized relaxation factor as a degradation invariant rather than the classical normalized relaxation curve but it changes the formalism and the model would become less easy to fit.

\section{References}

[1] J.K. Tessmar and A.M. Göpferich. Customized peg-derived copolymers for tissue-engineering applications. Macromolecular Bioscience, 7(1):23-39, 2007.

[2] J. S. Soares, James E. Moore Jr., and K. R. Rajagopal. Constitutive framework for biodegradable polymers with applications to biodegradable stents. Asaio Journal, 54(3):295-301, 2008.

[3] I.M. Ward and J. Sweeney Mechanical properties of solid polymers. Wiley, 2012.

[4] A.C. Pipkin and T.G. Rogers. A non-linear integral representation for viscoelastic behaviour. Journal of the Mechanics and Physics of Solids, 16(1):59 - 72, 1968.

[5] Y.C. Fung. Biomechanics: mechanical properties of living tissues. Springer Science \& Business Media, 2013.

[6] S.D. Abramowitch. An Improved Method to Analyze the Stress Relaxation of Ligaments Following a Finite Ramp Time Based on the Quasi-Linear Viscoelastic Theory. Journal of Biomechanical Engineering, 126(1):92 $-97,2004$.

[7] A. Nekouzadeh, K.M. Pryse, E.L. Elson, G.M. Genin. A simplified approach to quasi-linear viscoelastic modeling Journal of Biomechanics, 40(14):3070 - 3078, 2007.

[8] A. Göpferich. Mechanisms of polymer degradation and erosion. Biomaterials, 17(2):103 - 114, 1996.

[9] H. Tsuji. Autocatalytic hydrolysis of amorphous-made polylactides : effects of 1 -lactide content , tacticity , and enantiomeric polymer blending. Polymer, 43(6):1789-1796, 2002.

[10] T. Karjalainen, M. Hiljanen-Vainio, M. Malin, and J. Seppala. Biodegradable lactone copolymers. III. Mechanical properties of c-paprolactone and lactide copolymers after hydrolysis in vitro. Journal of Applied Polymer Science, 59(8):1299-1304, 1996.

[11] A. Leroy, C. Pinese, C. Bony, X. Garric, D. Noël, B. Nottelet, and J. Coudane. Investigation on the properties of linear PLA-poloxamer and star PLA-poloxamine copolymers for temporary biomedical applications. Materials science $\mathcal{E}$ engineering. C, Materials for biological applications, 33(7):4133-4139, 2013.

[12] Y. Wang, J. Pan, X. Han, C. Sinka, and L. Ding. A phenomenological model for the degradation of biodegradable polymers. Biomaterials, 29(23):3393-3401, 2008.

[13] L. Ding, R.L. Davidchack, and J. Pan. A molecular dynamics study of Young's modulus change of semicrystalline polymers during degradation by chain scissions. Journal of the mechanical behavior of biomedical materials, 5(1):224-230, 2012.

[14] H. Samami and J. Pan. A constitutive law for degrading bioresorbable polymers Journal of the mechanical behavior of biomedical materials, 59:430-445, 2016.

[15] A.C. Vieira, J.C. Vieira, J.M. Ferra, F.D. Magalhães, R.M. Guedes, and A.T. Marques. Mechanical study of PLA-PCL fibers during in vitro degradation. Journal of the mechanical behavior of biomedical materials, 4(3):451-460, 2011.

[16] A. Muliana and K.R. Rajagopal. Modeling the response of nonlinear viscoelastic biodegradable polymeric stents. International Journal of Solids and Structures, 49(7-8):989-1000, 2012.

[17] A.C. Vieira, R.M. Guedes, and V. Tita. Constitutive modeling of biodegradable polymers: Hydrolytic degradation and time-dependent behavior. International Journal of Solids and Structures, 51(5):1164-1174, 2014.

[18] Q. Breche, G. Chagnon, G. Machado, E. Girard, B. Nottelet, X. Garric and D. Favier. Mechanical behaviour?s evolution of a PLA-b-PEG-b-PLA triblock copolymer during hydrolytic degradation. Journal of the Mechanical Behavior of Biomedical Materials, 60:288-300, 2016.

[19] I Grizzi, H Garreau, S Li, and M Vert. Hydrolytic degradation of devices based on poly(DL-lactic acid) sizedependence. Biomaterials, 16(4):305-311, 1995.

[20] J.J. Sarver, P.S. Robinson, D.M. Elliott. Methods for Quasi-Linear Viscoelastic Modeling of Soft Tissue: Application to Incremental Stress-Relaxation Experiments. Journal of Biomechanical Engineering, 125(5):754$758,2003$.

[21] J. Lemaitre and J-L. Chaboche. Mécanique des matériaux solides. Dunod, Paris, 1985. 